\title{
Neumonitis lúpica: manejo con oxigenoterapia de alto flujo y posición prono. Reporte de caso y revisión de la literatura
}

\author{
Raymundo Flores-Ramírez, ${ }^{*}$ Jardiel Argüello-Bolaños, ${ }^{\ddagger}$ Kenia González-Perales, ${ }^{\S}$ \\ José Roberto Gallardo-Soberanis," Mónica Elizabeth Medina-Viramontes," \\ Karen Pamela Pozos-Cortés," Raúl Arturo González-Toribio," Julio Hiram López-Castro,"

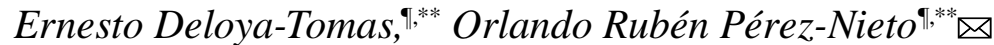

\footnotetext{
*Hospital de Especialidades «5 de Mayo», ISSSTEP Puebla; ${ }^{\ddagger}$ Hospital PEMEX Salamanca, Guanajuato;

${ }^{\S}$ Hospital General de Zona No. 2 IMSS, Monterrey, NL.; "Unidad Médica de Alta Especialidad IMSS, Puebla; `Hospital General San Juan del Río; **Miembros del Grupo AVENTHO para la investigación de la ventilación mecánica.
}

\begin{abstract}
RESUMEN. La neumonitis lúpica es una manifestación inusual del lupus eritematoso sistémico. Se presenta de forma inicial en 1-12\% de los casos, cursando con síndrome de insuficiencia respiratoria aguda y una evolución desfavorable con una mortalidad reportada desde $50 \%$ hasta 90\%. Existen estrategias para el manejo de pacientes con síndrome de insuficiencia respiratoria aguda grave, como la posición prono en pacientes ventilados mecánicamente asociada a medidas de protección alveolar. Recién se ha planteado la utilidad de la posición prono en pacientes despiertos con oxigenoterapia de alto flujo como una opción terapéutica. Presentamos el caso de una mujer de 19 años con neumonitis lúpica como debut de lupus eritematoso sistémico e insuficiencia respiratoria que fue diagnosticada y manejada con éxito con oxigenoterapia de alto flujo y posición prono, aunada al tratamiento con corticoesteroide, evitando el manejo avanzado de la vía aérea y ventilación mecánica.
\end{abstract}

Palabras clave: Lupus eritematoso sistémico, neumonitis lúpica, posición prono, oxigenoterapia de alto flujo, síndrome de insuficiencia respiratoria aguda.

\section{Lupus pneumonitis: management with high-flow oxygen therapy and prone position. Case report and review}

ABSTRACT. Lupus pneumonitis is an unusual manifestation of systemic lupus erythematosus. It's presented initially in 1-12\% of the cases manifested by acute respiratory distress syndrome (ARDS) and an unfavorable evolution with a mortality reported from $50 \%$ to $90 \%$. There are strategies for the management of patients with severe ARDS, such as the prone position in mechanically ventilated patients associated with alveolar protection measures. Recently, the usefulness of the prone position in patients with high-flow oxygen therapy has been proposed as a therapeutic option. We present the case of a 19-year-old woman with lupus pneumonitis as the debut of systemic lupus erythematosus and respiratory failure that was successfully diagnosed and managed with high-flow oxygen therapy and prone position, combined with corticosteroid treatment, avoiding advanced management of the airway and mechanical ventilation.

Key words: Systemic lupus erythematosus, lupus pneumonitis, prone position, high flow oxygen therapy, acute respiratory distress syndrome.

\section{INTRODUCCIÓN}

La neumonitis lúpica (NL) es una manifestación inusual del lupus eritematoso sistémico (LES). Se presenta de forma inicial en 1-12\% de los casos, cursando con sín-

\section{$\triangle$ Autor para correspondencia:}

Dr. Orlando Rubén Pérez-Nieto, Hospital General San Juan del

Río, Querétaro, México.

Correo electrónico: orlando_rpn@hotmail.com

Trabajo recibido: 21-XI-2018; aceptado: 28-II-2019 drome de insuficiencia respiratoria aguda (SIRA) y una evolución desfavorable con una mortalidad reportada desde $50 \%$ hasta $90 \% .1$

Existen estrategias para el manejo de paciente con SIRA grave como la posición prono asociada a medidas de protección alveolar. Recién se ha planteado la utilidad de la posición prono en pacientes despiertos con oxigenoterapia de alto flujo como una opción terapéutica alterna al manejo avanzado de vía aérea, esto basándose en la anatomía y fisiología pulmonar, ya que al encontrarse en posición prono se incrementa el volumen pulmonar al final de la espiración (VPFE), se 
retira el peso del corazón sobre las áreas pulmonares y se logra una distribución más homogénea del tamaño y de la ventilación alveolar favoreciendo el reclutamiento alveolar de zonas colapsadas. ${ }^{2}$

\section{ANTECEDENTES}

La NL es una forma de lesión pulmonar aguda manifestada principalmente por daño alveolar difuso, formación de membranas hialinas e infiltración de células mononucleares, manifestación poco frecuente del LES pero cuya importancia radica en su alta tasa de mortalidad, ya que en esta entidad además de la inflamación pleural, que es común en los pacientes con LES, también se encuentra compromiso pulmonar parenquimatoso. Se ha reportado que la mortalidad de los casos de LES con NL como manifestación inicial supera el $50 \%{ }^{1}$

La presentación clínica tiende a ser no específica, pero se caracteriza por tos productiva o seca, dolor pleurítico, fiebre, síntomas constitucionales, disnea y en ocasiones hemoptisis. A la exploración física pueden encontrarse estertores crepitantes, diminución del murmullo pulmonar, derrame pleural unilateral o bilateral e hipoxemia, la cual puede llegar a ser grave. ${ }^{1}$ Esta patología puede progresar rápidamente a insuficiencia respiratoria aguda que amerite ventilación mecánica (VM). ${ }^{3}$ Es importante sospechar el diagnóstico a la brevedad posible, teniendo en cuenta dos factores importantes: siempre en la evaluación inicial se debe descartar un proceso infeccioso bacteriano, ya que éste es una de las causas más frecuentes de afectación pulmonar en los pacientes con LES; ${ }^{4,5}$ y segundo, se debe considerar que la NL no necesariamente se correlaciona con los índices de actividad lúpica (SLEDAI, MEX-SLEDAI) lo que suele retrasar el diagnóstico.

No existe un estándar clínico para el diagnóstico de NL, casi siempre se realiza a través de la clínica/ inmunología compatible con LES ${ }^{6}$ más una tomografía computada de tórax con infiltración alveolar que no corresponda a un foco infeccioso bacteriano o viral. En ocasiones puede requerirse una biopsia pulmonar, cuya mayor utilidad radica en descartar otras patologías, ya que en esta entidad el estudio histológico es inespecífico, puede encontrarse inflamación, necrosis alveolar, capilaritis y hemorragia alveolar., ${ }^{1,7}$ Así, el diagnóstico suele ser realizado por exclusión en pacientes que no responden de manera empírica al tratamiento antimicrobiano y que presentan características extrapulmonares de LES como el eritema malar. ${ }^{1}$

Las recomendaciones de tratamiento están basadas en opiniones de expertos y reportes de casos. Se ha sugerido iniciar terapia antimicrobiana de amplio espectro dependiendo del grado de inmunosupresión y la probabilidad de un agente infeccioso en específico individualizando a cada paciente. El pilar del tratamiento es a base de pulsos de corticoesteroide a dosis altas (500 a 1,000 mg de metilprednisolona vía intravenosa cada 24 horas) por 3 días y posteriormente terapia inmunosupresora, principalmente con ciclofosfamida, azatioprina o micofenolato. Incluso hay algunos reportes de rituximab e inmunoglobulina intravenosa, sin nivel de evidencia para estas últimas recomendaciones. ${ }^{3}$

\section{Oxigenoterapia de alto flujo}

La oxigenoterapia de alto flujo (OAF) consiste en aportar un flujo de oxígeno, solo o mezclado con aire, por encima del flujo pico inspiratorio del paciente a través de una cánula nasal, ${ }^{8}$ cuenta con tres mecanismos de acción que pueden ayudar a mejorar la insuficiencia respiratoria del paciente: ${ }^{9}$

1) Aumento de la fracción inspirada de oxígeno $\left(\mathrm{FiO}_{2}\right)$ La concentración de oxígeno inspirada llega a ser mucho mayor que la otorgada por mecanismo de oxigenoterapia convencional de bajo flujo y casi cercana al $100 \%$.

2) Presión continua en la vía aérea (CPAP)

El flujo de oxígeno elevado genera una presión continua en la vía aérea, aumenta la capacidad residual funcional, disminuye las atelectasias y mejora la relación ventilación-perfusión, lo que puede aumentar la distensibilidad pulmonar y disminuir el trabajo respiratorio, contrarrestando incluso la presión positiva al final de la expiración (PEEP, del inglés positive end-expiratory pressure) intrínseca que se genera en pacientes con atrapamiento aéreo.

3) Confort del paciente

El oxígeno nasal cuando es calentado y humidificado se tolera mejor en especial a flujos elevados.

\section{Posición prono}

La posición prono fue descrita por primera vez en los años 70 en dos estudios, uno realizado por Bryan, ${ }^{10}$ quien destacó su potencial impacto favorable en la mecánica pulmonar; y otro realizado por Piehl et al. ${ }^{11}$ quienes reportaron una mejoría importante en la oxigenación. Un tercer trabajo de Douglas, ${ }^{12}$ publicado en 1977, planteó los beneficios hipotéticos de la posición prono; sin embargo, como sucede al inicio de nuevas investigaciones pese a la mejoría evidenciada en estos estudios, al no poseer un impacto estadístico significativo fueron pasados por alto sin provocar un impacto en la comunidad científica. 
Durante el SIRA se genera una alteración difusa de la permeabilidad vascular, hay edema y aumento del peso pulmonar, lo que favorece el desarrollo de atelectasias debido a la transmisión vertical de las fuerzas gravitatorias que comprimen las regiones pulmonares más dependientes, a esto se le suma el peso del corazón sobre el parénquima pulmonar y el efecto de la masa abdominal, que en posición supina comprime en dirección cefálica las partes posteriores del diafragma y el parénquima pulmonar adyacente. La parálisis diafragmática por el uso de bloqueadores neuromusculares, la disminución del líquido surfactante pulmonar y la distensión abdominal pueden agravar este problema. ${ }^{13}$

La posición prono tiene la ventaja de aumentar la capacidad residual funcional al reclutar alvéolos ocupados en zonas dependientes por modificación del eje esterno-vertebral y retira el peso del corazón sobre el parénquima pulmonar, en este caso en particular el derrame pericárdico podría contribuir aún más al colapso alveolar en la zona pulmonar ubicada por debajo de este órgano. ${ }^{13}$

En 2013, un estudio francés multicéntrico demostró por primera vez un beneficio significativo sobre la tasa de mortalidad a los 28 días: $16 \%$ en el grupo con posición prono (38/237 pacientes) y $32.8 \%(75 / 229$ pacientes) en el grupo de control ( $p<0.0001)$, cabe señalar que los pacientes incluidos tenían una $\mathrm{PaO}_{2} /$ $\mathrm{FiO}_{2}<150 \mathrm{mmHg}$ y fueron manejados con $\mathrm{VM}$ protectora y bloqueo neuromuscular. ${ }^{14} \mathrm{~A}$ partir de esta fecha, el uso de la posición prono es cada vez más frecuente y difundido a nivel global. ${ }^{15}$

\section{Posición prono combinada con OAF o VN}

Teóricamente, los beneficios de la posición prono pueden afectar también a los pacientes con respiración espontánea, en los cuales podría contribuir al éxito de la estrategia no invasiva. Los datos relativos a la aplicación de la posición prono en dicho contexto se limitan a pequeñas series de casos y un estudio retrospectivo de 15 pacientes no intubados con insuficiencia respiratoria aguda donde tres pacientes (20\%) recibieron sedación leve sin ajustes de dosis durante el curso del estudio, la duración media de la posición prona fue de tres horas y sólo en dos casos (5\%) la pronación tuvo que interrumpirse después de 30 minutos debido a la incomodidad del paciente, mientras que ninguna otra complicación respiratoria o técnica, como el desplazamiento de catéteres permanentes, edema facial, úlceras por presión, neuropatías por presión, compresión de los nervios y vasos retinianos o vómitos fue documentada. Dada su alta tolerabilidad, simplicidad de uso y efecto positivo en la comodidad de los pacientes cuando se comparan tanto con la VN como con el flujo de oxígeno administrado a través de una máscara facial, la OAF puede ser la estrategia óptima para el manejo respiratorio de los pacientes con respiración espontánea durante la posición prono. ${ }^{16}$

Un estudio español con una muestra de 20 pacientes sanos demostró un aumento del VPFE y una disminución en la frecuencia respiratoria (FR) usando OAF y relacionándolo con los cambios de posición corporal (prono y supino) sin presentar efectos adversos, donde evidenciaron mediante tomografía por impedancia eléctrica que en posición prono el VPFE presentó una distribución más homogénea que en posición supino. ${ }^{2}$

Hasta el momento, en nuestro país se ha descrito sólo un caso de un paciente con atelectasia y neumotórax en posición prono con ventilación espontánea y OAF, sin complicaciones reportadas y en quien se evitó la VM. ${ }^{17}$

Al presente, se encuentra en fase de reclutamiento un estudio piloto de viabilidad (Optiprone) para evaluar la seguridad y eficacia de la posición prono combinada con OAF en pacientes con SIRA que tengan un índice $\mathrm{PaO}_{2} / \mathrm{FiO}_{2}<200$, se espera reclutar 15 pacientes y tener resultados para finales del 2019. ${ }^{18}$
Figura 1:

Imagen comparativa de cortes axiales tomográficos al ingreso y posterior a 7 días de tratamiento.
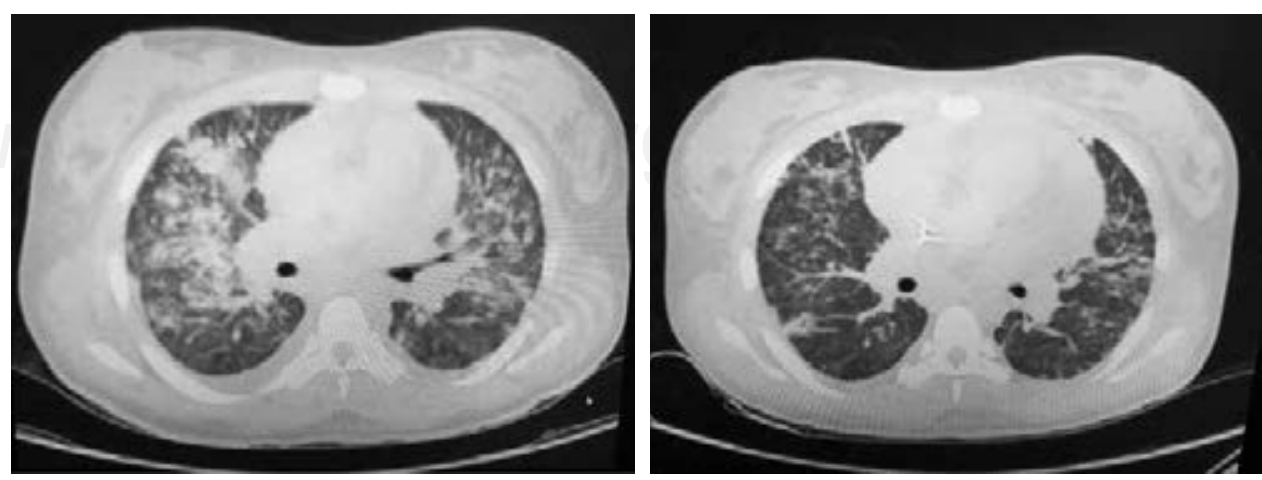


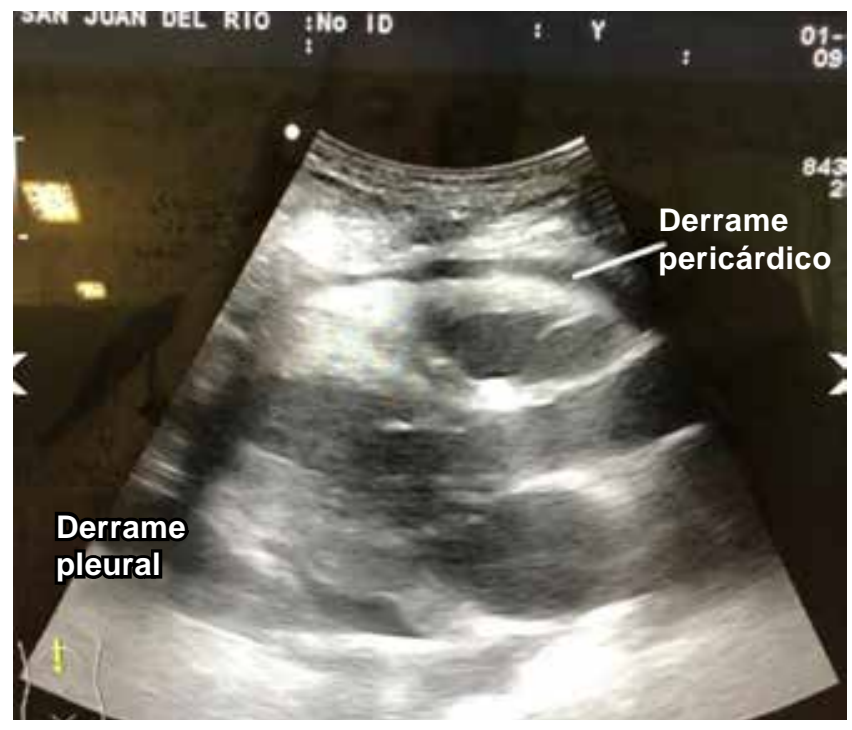

Figura 2: Ventana ultrasonográfica subxifoidea donde se observa derrame pericárdico y derrame pleural izquierdo.

\section{DESCRIPCIÓN DEL CASO}

Mujer de 19 años de edad con antecedente de púrpura trombocitopénica hace cuatro años tratada con prednisona, finalizando esquema terapéutico un año antes aproximadamente sin recurrencias, sin consumo crónico de otros fármacos, sin antecedentes heredofamiliares relevantes. Inició su padecimiento actual un mes previo a su ingreso con cuadro de infección de vías respiratorias altas, tratada con antibióticos sin presentar mejoría. Se documentó hemoglobina de $10.5 \mathrm{mg} / \mathrm{dl}$, fiebre de $39{ }^{\circ} \mathrm{C}$ y tos productiva con secreción hialina acompañada de síntomas constitucionales.

Ingresó al Servicio de Urgencias de nuestro hospital por presentar disnea, exacerbación de la tos, con expectoración hialina, cefalea y vómito. A su ingreso se encuentra con los siguientes signos vitales: tensión arterial (TA) 85/36 mmHg, frecuencia cardíaca (FC) 130 latidos por minuto, FR 36 respiraciones por minuto, temperatura $37.5^{\circ} \mathrm{C}$, SatO ${ }_{2}$ por oximetría de pulso de $80 \%$. Se encontró despierta, orientada, deshidratada, con eritema malar, lesiones eritematosas que desaparecen a la digitopresión en antebrazos y codos, áreas pulmonares con estertores crepitantes bilaterales de predominio subescapular, precordio rítmico, rápido, sin agregados.

En los estudios de laboratorio se documentó a su ingreso una hemoglobina $(\mathrm{Hb})$ de $6.3 \mathrm{~g} / \mathrm{dl}$, plaquetas $380 \times 10^{3} \mu \mathrm{l}$, leucocitos $5.19 \times 10^{3} \mu \mathrm{l}$ con linfopenia de $0.68 \times 10^{3} \mu \mathrm{l}$, gasometría con $\mathrm{pH} 7.35, \mathrm{PCO}_{2} 20 \mathrm{mmHg}$, $\mathrm{PO}_{2} 55 \mathrm{mmHg}, \mathrm{HCO}_{3} 16 \mathrm{mmol} / \mathrm{l}, \mathrm{SatO}_{2} 77 \%$, lactato
$1.8 \mathrm{mmol} / \mathrm{l}$, déficit de base de -7. Electrolitos, pruebas de funcionamiento hepático, química sanguínea dentro de parámetros de referencia. TAC de tórax con vidrio esmerilado, broncograma aéreo, imágenes de densidad aumentada en el parénquima pulmonar sugerente de ocupación alveolar y derrame pleural bilateral (figura 1). Se realizó rastreo ultrasonográfico encontrando un patrón pulmonar tipo B con colas de cometa hasta cinco por campo, con signo de sinusoide bilateral correspondiente a derrame pleural bilateral de aproximadamente $380 \mathrm{ml}$ en lado derecho y $240 \mathrm{ml}$ en lado izquierdo, también se observó derrame pericárdico en ventana subxifoidea de aproximadamente $180 \mathrm{ml}$ (figura 2).

Se inició protocolo diagnóstico y terapéutico de neumonía atípica con oseltamivir, levofloxacino, toma de cultivos y PCR para virus de la influenza. Se realizó transfusión de concentrados eritrocitarios, reposición hídrica guiada por monitoreo de gasto cardíaco estimado no invasivo (esCCO) y ultrasonografía de vena yugular y cava inferior. Dado que persistió con datos de choque distributivo requirió norepinefrina en infusión. El deterioro respiratorio aumentó presentando taquipnea con FR de 35 a 40 respiraciones por minuto, disnea, diaforesis y taquicardia hasta 160 latidos por minuto.

Se inició manejo con OAF programado a $60 \mathrm{l} / \mathrm{min}$ y una fracción inspirada de oxígeno de $60 \%$ con lo que se logró mejoría parcial; sin embargo, en días subsecuentes presentó aumento de disnea, taquipnea e hi-

Tabla 1: Parámetros clínico/gasométricos previos, durante y posteriores a la terapia combinada de posición prono y cánulas de alto flujo.

\begin{tabular}{|l|l|l|l|}
\hline $\begin{array}{c}\text { Parámetros } \\
\text { clínicos y } \\
\text { gasométri- } \\
\text { cos }\end{array}$ & $\begin{array}{c}\text { Oxigenote- } \\
\text { rapia de alto } \\
\text { flujo } \\
(60 \mathrm{l} / \mathrm{min}, \\
\left.\mathrm{FiO}_{2} 60 \%\right)\end{array}$ & $\begin{array}{c}\text { Posición } \\
\text { prono + oxi- } \\
\text { genoterapia } \\
\text { de alto flujo }\end{array}$ & $\begin{array}{c}\text { Supinación } \\
\text { + oxigenote- } \\
\text { rapia de alto } \\
\text { flujo* }\end{array}$ \\
\hline $\mathrm{FR}$ & $38 \mathrm{rpm}$ & $30 \mathrm{rpm}$ & $24 \mathrm{rpm}$ \\
\hline $\mathrm{FC}$ & $150 \mathrm{lpm}$ & $123 \mathrm{lpm}$ & $110 \mathrm{lpm}$ \\
\hline $\mathrm{TA}$ & $116 / 51$ & $123 / 60$ & $118 / 58$ \\
$\mathrm{mmHg}$ & $\mathrm{mmHg}$ & $\mathrm{mmHg}$ \\
\hline $\mathrm{SatO}$ & $90 \%$ & $99 \%$ & $93 \%$ \\
\hline $\mathrm{pH}_{2}$ & 7.35 & 7.36 & 7.40 \\
\hline $\mathrm{PaO}_{2}$ & $69 \mathrm{mmHg}$ & $90 \mathrm{mmHg}$ & $81 \mathrm{mmHg}$ \\
\hline $\mathrm{PaCO}_{2}$ & $20 \mathrm{mmHg}$ & $26 \mathrm{mmHg}$ & 30.4 \\
\hline $\mathrm{PaO}_{2} / \mathrm{FiO}_{2}$ & 115 & 300 & 270 \\
\hline
\end{tabular}

FR: frecuencia respiratoria; FC: frecuencia cardíaca; TA: tensión arterial; $\mathrm{SatO}_{2}$ : saturación de oxígeno por oximetría de pulso; $\mathrm{PaO}_{2}$ : presión parcial de oxígeno arterial; $\mathrm{PaCO}_{2}$ : presión parcial de dióxido de carbono arterial; $\mathrm{FiO}_{2}$ : fracción inspirada de oxígeno.

*Después de 3 días de pronación intermitente + oxígeno terapia de alto flujo. En este momento ya sólo con $\mathrm{FiO}_{2}$ al $30 \%$. 


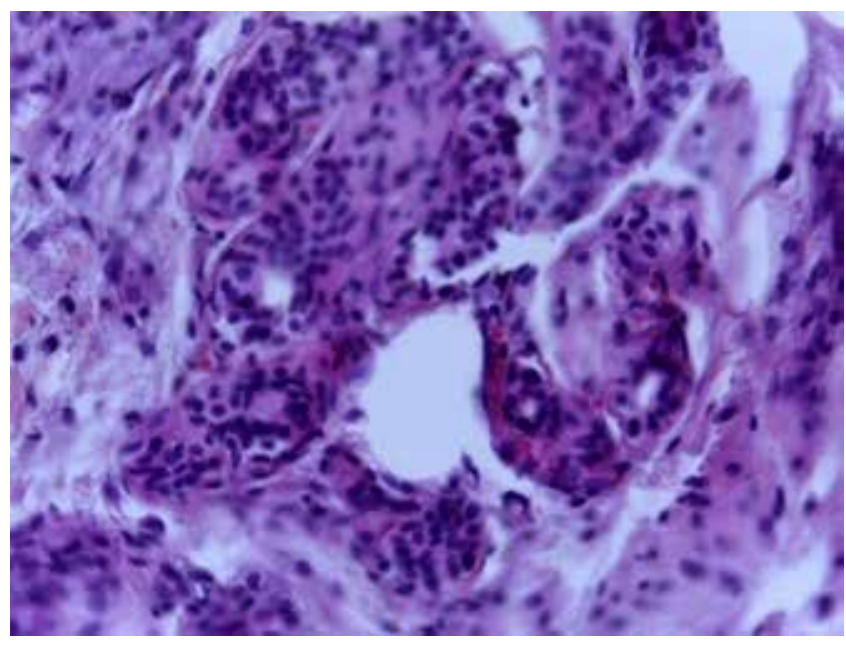

Figura 3: Fotografía tomada al microscopio de un corte de biopsia incisional de piel gruesa, en donde se observa vasculitis de vasos arteriolares de pequeño calibre situados preferentemente en la dermis profunda y tejido subcutáneo adiposo. Infiltrado de tipo linfocitario.

poxemia, por lo que se decidió pronar a la paciente con ventilación espontánea sumado a OAF con la finalidad de incrementar la capacidad residual funcional y liberar el peso generado por el corazón sobre el parénquima pulmonar. Con esta maniobra se logró un cambio inmediato en la mecánica ventilatoria y en los parámetros gasométricos (tabla 1).

Se mantuvo sin sedación, en posición prono de forma intermitente durante 3 días, entre 3 y 6 horas por día de acuerdo con la tolerancia de la paciente. Durante el resto de su hospitalización no presentó datos clínicos o bioquímicos de que requiriera $\mathrm{VM}$.

En controles posteriores la linfopenia se acentuó hasta $0.25 \times 10^{3} \mu \mathrm{l}$, la procalcitonina se mantuvo entre $0.50-0.60 \mathrm{ng} / \mathrm{ml}$ descartando un proceso infeccioso bacteriano, se descartó neumonía por virus de la influenza con PCR negativa. El reporte de perfil inmunológico mostró hipocomplementemia, anticuerpos antinucleares (ANA) 1:640, anti-DNA de doble cadena $480 \mathrm{UI} / \mathrm{ml}$, anti-Sm negativo, p-ANCA y c-ANCA negativos; además, se realizó biopsia de lesiones cutáneas en brazo derecho confirmando vasculitis (figura 3 ). Con todo lo anterior se reunieron 6 criterios del Colegio Americano de Reumatología (ACR, por sus siglas en inglés) y 9 criterios del Grupo de Clínicas Colaborativas Internacionales del Lupus Sistémico (SLICC, por sus siglas en inglés) para clasificar como LES. ${ }^{5}$

Debido a la alta sospecha diagnóstica de LES y la insuficiencia respiratoria grave sin foco infeccioso determinado, se iniciaron pulsos de metilprednisolona a dosis de $1 \mathrm{~g}$ intravenoso cada $24 \mathrm{~h}$ por tres dosis y posteriormente $50 \mathrm{mg}$ de prednisona vía oral cada $24 \mathrm{~h}$. La paciente presentó mejoría paulatina de la sintomatología, disminuyó el derrame pleural derecho a $200 \mathrm{ml}$, el izquierdo a $140 \mathrm{ml}$, el derrame pericárdico persistió, los infiltrados tomográficos alveolares disminuyeron, se logró retiro de la oxigenoterapia de alto flujo para pasar a oxigenoterapia de bajo flujo (3 l/min).

La paciente egresó de la Unidad de Cuidados Intensivos para continuar protocolo y manejo en el Servicio de Medicina Interna donde posteriormente se retiró el oxígeno suplementario.

\section{DISCUSIÓN}

La NL es una entidad patológica poco frecuente, sin un estándar diagnóstico definitivo, pero con una alta tasa de mortalidad, en especial cuando se presenta como manifestación inicial del LES. Al manifestarse como un SIRA en un paciente con compromiso inmunológico se debe descartar un padecimiento infeccioso a la brevedad posible, es recomendable la toma de cultivos de expectoración y puede considerarse la toma de hemocultivos, así como solicitar PCR para virus respiratorios. En caso de tener pruebas negativas o que el curso clínico no corresponda a un proceso infeccioso, esto nos acerca más al diagnóstico de NL que se suele realizar por exclusión.

EI SIRA grave de cualquier causa incrementa notablemente la mortalidad en los pacientes, hasta el momento el protocolo terapéutico básicamente consiste en VM con volúmenes tidales bajos ( $6 \mathrm{ml} / \mathrm{kg}$ peso predicho), presiones bajas (presión de distensión alveolar $<13 \mathrm{cmH}_{2} \mathrm{O}$, presión meseta $<24 \mathrm{cmH}_{2} \mathrm{O}$ ), bloqueo neuromuscular, posición prono, sedoanalgesia óptima y todas las demás medidas de un paciente crítico (nutrición temprana, rehabilitación temprana, etc.). Aunque recién se ha planteado el beneficio de la posición prono en paciente despierto con VN, específicamente con cánulas de alto flujo. En nuestro caso se optó por realizar esta maniobra en nuestra paciente, con lo cual logramos evitar la necesidad de VM y disminuir los riesgos que ésta conlleva.

Es importante mencionar que esta terapia (prono + OAF) se sugiere como una alternativa a la intubación y VMI en el contexto de un paciente con SIRA, en quien fracasa la VN y se debe realizar con todo el equipo preparado para el abordaje avanzado de la vía aérea y conexión al ventilador mecánico de ser necesario. No hay un estándar de tiempo para mantener esta posición, pero recomendamos que al menos sea de 2 a 6 horas alternando con 2 horas de supinación para evitar la fatiga del paciente. En nuestra experiencia la 
mejoría es casi inmediata, observando un aumento de la $\mathrm{SatO}_{2}$ por oximetría de pulso, diminución de la FR, mejoría en la FC; y muy importante, el paciente expresó una mejoría en la disnea.

Al día de hoy no existe evidencia suficiente para recomendar la utilización de la posición prono en pacientes en ventilación espontánea y, teóricamente, podría generar situaciones de riesgo como retraso en el inicio de la VM; no obstante, tampoco se cuenta con información para desacreditar su uso. La tendencia actual a la menor invasividad y a la utilización de dispositivos no invasivos efectivos como la OAF pueden contribuir a mayor posibilidad de éxito en este tipo de pacientes.

\section{CONCLUSIÓN}

La posición prono combinada con OAF es una maniobra terapéutica con gran sustento fisiopatológico que podría evitar iniciar la VM en pacientes con SIRA moderadograve. Sin embargo, es necesario realizar estudios de seguridad y eficacia terapéutica y posteriormente aleatorizados para emitir una recomendación sobre su uso.

\section{Financiamiento}

Este artículo no recibió financiamiento de ningún tipo.

\section{Conflicto de intereses}

Todos los autores declaran no tener conflicto de intereses.

\section{REFERENCIAS}

1. Chattopadhyay B, Chatterjee A, MaitiA, Debnath NB. Systemic lupus erythematosus presenting as acute lupus pneumonitis in a young female. J Postgrad Med 2015;61(2):129-130. doi: 10.4103/0022-3859.150902.

2. Riera J, Pérez P, Cortés J, Roca O, Masclans JR, Rello J. Effect of high-flow nasal cannula and body position on end-expiratory lung volume: a cohort study using electrical impedance tomography. Respir Care 2013;58(4):589-596. doi: 10.4187/respcare.02086.

3. Kamen DL, Strange C. Pulmonary manifestations of systemic lupus erythematosus. Clin Chest Med 2010;31(3):479-488. doi: 10.1016/j.ccm.2010.05.001.

4. Pena GA, Rodríguez CP, González BFJ. Manifestaciones pulmonares del lupus eritematoso sistémico. Pneuma 2010;6(3):109-116.

5. Aguilera-Pickens G, Abud-Mendoza C. Pulmonary manifestations in systemic lupus erythematosus: pleural involvement, acute pneumonitis, chronic interstitial lung disease and diffuse alveolar hemorrhage. Reumatol Clin 2018;14(5):294-300. doi: 10.1016/j.reuma.2018.03.012.
6. Petri M, Orbai AM, Alarcón GS, et al. Derivation and validation of the systemic lupus international collaborating clinics classification criteria for systemic lupus erythematosus. Arthritis Rheum 2012;64(8):26772686. doi: 10.1002/art.34473.

7. Matthay RA, Schwarz MI, Petty TL, et al. Pulmonary manifestations of systemic lupus erythematosus: review of twelve cases of acute lupus pneumonitis. Medicine (Baltimore) 1975;54(5):397-409.

8. Pilar OFJ, López FYM. Oxigenoterapia de alto flujo. An Pediatr Contin 2014;12(1):25-29.

9. Nishimura M. High-flow nasal cannula oxygen therapy in adults. J Intensive Care 2015;3(1):15-23. doi: 10.1186/ s40560-015-0084-5.

10. Bryan AC. Conference on the scientific basis of respiratory therapy. Pulmonary physiotherapy in the pediatric age group. Comments of a devil's advocate. Am Rev Respir Dis 1974;110(6 Pt 2):143-144.

11. PiehI MA, Brown RS. Use of extreme position changes in acute respiratory failure. Crit Care Med 1976;4(1):13-14.

12. Douglas WW, Rheder K, Beynen FM, Sessler AD, Marsh HM. Improved oxygenation in patients with acute respiratory failure: the prone position. Am Rev Respir Dis 1977;115(4):559-566.

13. Hernández-López GD, Mondragón-Labelle T, TorresLópez L, Magdaleno-Lara G. Posición prono, más que una estrategia en el manejo de pacientes con síndrome de insuficiencia respiratoria aguda. Rev Hosp Jua Mex 2012;79(4):263-270.

14. Guérin C, Reignier J, Richard JC, et al.; PROSEVA Study Group. Prone positioning in severe acute respiratory distress syndrome. N Engl J Med 2013;368(23):21592168. doi: 10.1056/NEJMoa1214103.

15. Gattinoni L, Taccone P, Carlesso E, Marini JJ. Prone position in acute respiratory distress syndrome. Rationale, indications, and limits. Am J Respir Crit Care Med 2013;188(11):1286-1293. doi: 10.1164/rccm.201308$1532 \mathrm{Cl}$.

16. Scaravilli V, Grasselli G, Castagna L, et al. Prone positioning improves oxygenation in spontaneously breathing nonintubated patients with hypoxemic acute respiratory failure: a retrospective study. J Crit Care 2015;30(6):1390-1394. doi: 10.1016/j. jcrc.2015.07.008.

17. Lacorte-Hernández $\mathrm{CL}$, Zamarrón-López El, SorianoOrozco R, Deloya-Tomas E, Pérez-Nieto OR. Nonconventional management of bilateral pneumothorax and pulmonary contusion with high-flow oxygen therapy and prone position. Int J Recent Sci Res 2018;9(10):2927029274. doi.org/10.24327/ijrsr.2018.0910.2826

18. ClinicalTrials.gov [Internet]. Massimo Antonelli (MD): National Library of Medicine (US) 2017 Mar 29. Identifier NCT03095300. Prone position in during high flow oxygen therapy in acute hypoxemic respiratory failure (Optiprone); 2017. Access date: 2018 December 19. Available from: https://clinicaltrials.gov/ct2/show/ NCT03095300. 